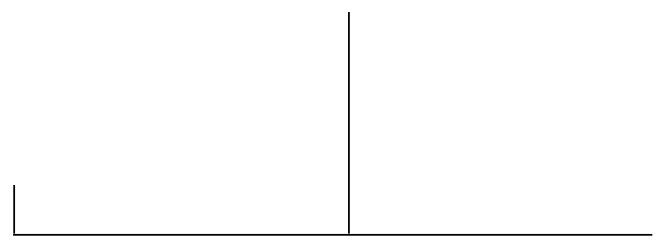

Rev. Latinoam. Psicopat. Fund., São Paulo, v. 13, n. 2, p. 318-332, junho 2010

\title{
A paranoia em 1904 - uma etapa na construção nosológica de Emil Kraepelin
}

Ana Maria Galdini Raimundo Oda

Este artigo apresenta o texto de Kraepelin sobre a paranoia, extraído da sétima edição de seu tratado de psiquiatria (1904), traduzido para o português e publicado pela primeira vez em 1905, na revista Arquivos Brasileiros de Psiquiatria, Neurologia e Ciências Afins. Indica-se a posição desta categoria na nosologia de Kraepelin, bem como se discute aspectos da difusão de suas ideias no Brasil.

Palavras-chave: Emil Kraepelin, paranoia - história, nosologia psiquiátrica - história, transtornos delirantes - história, Arquivos Brasileiros de Psiquiatria, Neurologia e Ciências Afins 


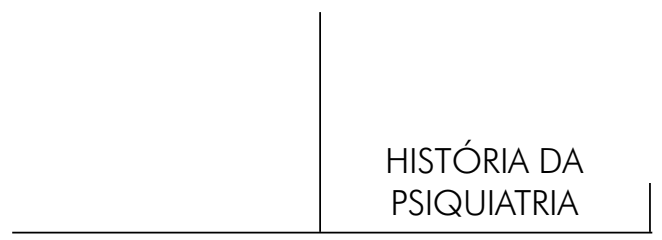

Emil Kraepelin

Em 1886, o médico alemão Emil Kraepelin (1856-1926) iniciava sua carreira docente na Imperial Universidade russa de Dorpat. Em sua conferência inaugural, discutia as vertentes das pesquisas psiquiátricas coetâneas, observando que a este setor da medicina faltava ainda um idioma científico comum, de forma que muitas vezes os psiquiatras se viam sofrendo do mesmo mal que confundira os construtores da mítica Torre de Babel (Kraepelin, 1887).

A percepção da necessidade de uma linguagem que permitisse a comunicação entre as várias "escolas psiquiátricas" resultou no precoce estabelecimento do que se pode chamar de um programa fundamental de pesquisa, cujo objetivo era desenvolver descrições clínicas consistentes, passíveis de alocação em uma classificação estável das psicoses. Nesta empreitada, tendo Wilhelm Wundt (1832-1920) e Karl Kahlbaum (1828-1899) como referências teóricas basilares, ele buscou construir um suporte empírico para sua nosografia, que se define como uma categorização de base filosófica kantiana ${ }^{1}$ (Berrios e Hauser, 1988).

No decorrer de sua longa carreira de professor de psiquiatria, em Dorpat (1886-1890), Heidelberg (1891-1903) e Munique (1903-1922) (Shorter, 2005), Kraepelin desenvolveu esse programa de pesquisa. Em 1922, aposentando-se da Universidade de Munique, passou a dedicar-se exclusivamente ao Instituto Alemão de Pesquisas Psiquiátricas - Deutsche Forschungsanstalt für Psychiatrie - criado em 1917 (Moreira, 1926).

A análise da trajetória profissional de Kraepelin mostra seu interesse por vários métodos de pesquisa clínica que, de acordo com Eric Engstrom (2007), se articulam no decorrer de sua carreira: em Dorpat, a utilização de testes psi-

1. Como explica Jorge Saurí (2001, p. 20), Kraepelin é um dos que considera que "diagnosticar, além de consistir na observação, é esquematizar por meio de uma apresentação mediadora e catalogante", de acordo com o procedimento criticista kantiano. Assim, o objetivo da observação empírica é buscar indicadores semiológicos que sejam relevantes para cada categoria diagnóstica, a partir de regras já preestabelecidas. 


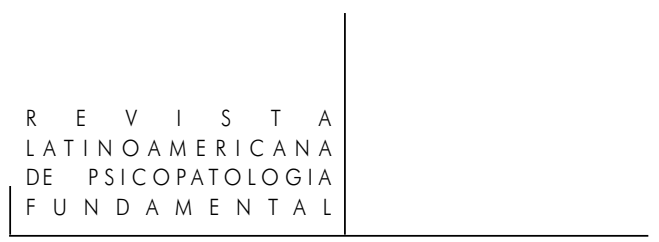

cológicos (seguindo Wundt); em Heidelberg, o desenvolvimento de estratégias de observação e documentação (usando as Zählkarten, coleções de registros sumarizados de casos, em formato semiestandardizado) e, em Munique, as investigações epidemiológicas mais amplas e o que se chamava então de higiene mental.

Graduado em medicina em 1878, Kraepelin trabalhou como alienista em vários serviços médicos alemães, ao mesmo tempo em que completava sua especialização em psiquiatria. Logo em 1883, publicou o seu Compendium der Psychiatrie (Compêndio de psiquiatria). A partir da segunda edição, em 1887, a obra passou a se chamar Psychiatrie: Ein Lehrbuch für Studirende und Aerzte (aproximadamente, Psiquiatria: um manual para estudantes e médicos) e teve nove edições revisadas e ampliadas (entre 1883 e 1927), sendo oito em vida do autor. $^{2}$

Especialmente desde a sexta edição (1899), o manual ou tratado de Kraepelin circulou amplamente nos meios psiquiátricos internacionais, no original ou por meio de traduções de partes selecionadas, publicadas como livros, ${ }^{3}$ ou ainda por meio de traduções de excertos mais breves.

\section{A difusão das ideias de Kraepelin no Brasil}

Tal é o caso do trecho que republicamos no presente número da Revista Latinoamericana de Psicopatologia Fundamental. Ele foi extraído da sétima edição (1904) de Psychiatrie, traduzido do alemão para o português por Antonio Austregésilo ${ }^{4}$ (1876-1960), médico do Hospício Nacional de Alienados, e publicado

2. Primeira edição: 1883 (Compendium der Psychiatrie); segunda edição: 1887 (Psychiatrie: Ein Lehrbuch...); terceira edição: 1889; quarta edição: 1893; quinta edição: 1896; sexta edição: 1899; sétima edição: 1903-1904 (2 volumes); oitava edição: 1909-1915 (4 volumes); nona edição: 1927, editada por Johannes Lange (Shorter, 2005, p. 156-157).

3. Por exemplo, a tradução para o inglês de parte da sétima edição alemã do Lehrbuch (1904), publicada com o título de Clinical Psychiatry (1907); e as traduções para o inglês de partes da oitava edição alemã (1913 e 1915), intituladas Dementia Praecox and Paraphrenia (1919) e Manic Depressive Insanity and Paranoia (1921).

4. Antonio Austregésilo Rodrigues Lima era pernambucano e formou-se pela Faculdade de Medicina do Rio de Janeiro (1899), onde mais tarde seria professor substituto de Clínica Médica (1909) e catedrático de Neurologia (1912). Participou da fundação dos Arquivos Brasileiros de Psiquiatria, Neurologia e Ciências Afins e dos Arquivos Brasileiros de Medicina. Poeta e ensaísta, foi eleito membro da Academia Brasileira de Letras (1914). Foi ainda deputado federal (1922 a 1930) (Teive e cols., 1999). Veja-se sua biografia no site da Academia Brasileira de Letras, disponível em: http://www.academia.org.br. 
no volume inicial dos Arquivos Brasileiros de Psiquiatria, Neurologia e Ciências Afins, sob o título de "Paranoia (Verrücktheit) - Prof. E. Kraepelin", em três partes, no decorrer do ano de 1905.

A revista Arquivos Brasileiros de Psiquiatria, Neurologia e Ciências Afins foi a primeira especializada na área em nosso país, fundada no Rio de Janeiro por Juliano Moreira (1873-1933) e Afrânio Peixoto (1876-1947). É importante notar que sua publicação fez parte dos empenhos em fortalecer científica e politicamente o nascente campo da psiquiatria brasileira, empreendidos por um grupo de médicos reunidos em torno de J. Moreira, diretor (entre 1903 e 1930) do Hospício de Alienados da capital federal, a mais importante instituição psiquiátrica nacional.

Escrito pelos editores, o artigo inaugural dos Arquivos também trata da paranoia, o que parece uma escolha significativa. Ao iniciar "A paranoia e as síndromes paranoides", ${ }_{5}^{5}$ Juliano Moreira e Afrânio Peixoto deploram a imprecisão do diagnóstico, pois "ora se descrevem doenças diversas com o mesmo nome, ora enfermidades idênticas com outros nomes, ora, finalmente, algumas designações qualificativas precisam espécies mórbidas que se não podem conter dentro do mesmo gênero. É um verdadeiro estado de Babel ou confusão psiquiátrica, em que a gente se arrisca, no fim, a não sair à luz com uma ideia definida" (Moreira e Peixoto, 1905a, p. 5).

Nesse artigo, em que a (in)definição da paranoia é exemplar do "estado de Babel" em que se encontra a psiquiatria, os autores brasileiros declaram abertamente sua filiação a Emil Kraepelin, dizendo que "são suas ideias que propagamos, procurando na descrição e no exemplo traçar o limite com os delírios expansivos e os de perseguição, (...) ou simplesmente síndromes paranoides, epifenômenos de quase todas as formas de doença mental" (Moreira e Peixoto, 1905a, p. 6). O volume do ano de 1905 dos Arquivos traz ainda as principais categorias da classificação kraepeliniana, descritas e comentadas (Moreira e Peixoto, 1905b). ${ }^{6}$ Desta forma, os editores defendem não só a concepção de paranoia de Kraepelin, mas, sobretudo, os princípios de seu arranjo nosológico.

5. O artigo foi republicado em 2001, na Revista Latinoamericana de Psicopatologia Fundamental, v. 4, n. 2, p. 134-167, jun.2001).

Duas versões deste trabalho foram apresentadas em comunicações, a primeira ao III Congresso Latinoamericano de Medicina de Buenos Aires (1904), segundo Peixoto (1911]), e a segunda em 1906, ao XV Congresso Internacional de Medicina de Lisboa, esta sob o título "La paranoïa légitime: son origine et nature" (Moreira, 1908; Peixoto, 1911]).

6. Nesse volume, há também os artigos 'A loucura maníaco-depressiva' (de Afrânio Peixoto) e "Sobre a psicose maníaco-depressiva" (de Francisco Franco da Rocha), e as resenhas de Antonio Austregésilo sobre os trabalhos "A concepção da paranóia", de Adelino Pinto e "A paranoia segundo Kraepelin”, de Ulysses Vianna Filho. 


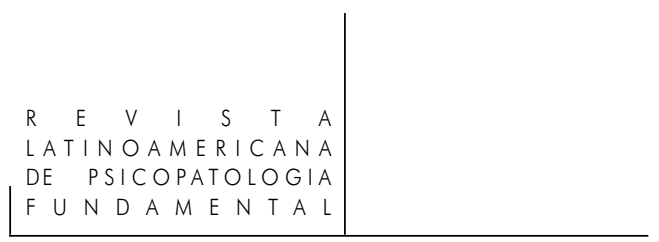

No decorrer das primeiras três décadas do século XX, Moreira e alguns de seus colaboradores seguiriam registrando o desenvolvimento das classificações de Kraepelin; no caso específico dos quadros delirantes (paranoia, parafrenia e demência precoce), o diretor do Hospício Nacional traduziu e comentou os novos arranjos nosológicos propostos pelo professor alemão, tão logo estes foram publicados (Moreira, 1919; Moreira, 1921), ${ }^{7}$ atentamente cuidando da tarefa de difusão das ideias de Kraepelin no Brasil.

Como se nota, Juliano Moreira tinha uma visão científica muito próxima à de Emil Kraepelin, sendo possível indicar várias correspondências entre as agendas de pesquisa de ambos; uma delas diz respeito ao interesse pelos estudos denominados de psiquiatria comparada. ${ }^{8} \mathrm{Na}$ análise de Engstrom (2007), o interesse de Kraepelin pelas manifestações das enfermidades mentais em diferentes sociedades deve ser compreendido como parte de sua abordagem epidemiológica, que visava comparar não só os sintomas clínicos, mas também o peso relativo de distintos fatores etiológicos, em contextos socioculturais diversos, com especial foco nas categorias dementia paralytica e dementia praecox.

Em perspectiva similar - no caso, discutindo as relações entre doença mental, raça, clima e meio sociocultural (leia-se, civilização e progresso) no Brasil Moreira produziu artigos e apresentou comunicações em congressos internacionais, tendo se tornado um autor de referência no assunto. ${ }^{9}$ Entre 1905 e 1906,

7. Assim, em "Classificações em medicina mental", Moreira (1919, p. 109-113) registra a classificação da $8^{a}$ edição alemã do tratado de Kraepelin (terminada em 1915); em "O novo agrupamento nosográfico das doenças mentais do prof. Emil Kraepelin” (Moreira, 1921, p. 184-188), refere-se àquela que consta na $4^{a}$ edição alemã de Einführung in die psychiatrische Klinik (Introdução à clínica psiquiátrica), de 1921.

8. O campo de interesses da chamada psiquiatria comparada (hoje, psiquiatria transcultural) não foi "fundado" por Kraepelin, mas já vinha se configurando historicamente antes de sua famosa viagem a Java, no contexto da medicina praticada no mundo colonial do século XIX, como buscamos indicar em outro trabalho (Oda, Dalgalarrondo e Banzato, 2005).

9. Para uma discussão deste tópico, veja-se Oda e Piccinini, 2005, onde se lista a produção de Moreira e colaboradores nesse campo. Destacamos dois trabalhos recentemente republicados: Moreira, J.; Peixoto, A. Les maladies mentales dans le climats tropicaux. Arquivos Brasileiros de Psiquiatria, Neurologia e Ciências Afins, v. 2, n. 1, p. 222-241, 1906. [Traduzido e publicado como: Moreira, J.; Peixoto, A. As doenças mentais nos climas tropicais. Revista Latinoamericana de Psicopatologia Fundamental, v. 8, n. 4, p. 794-811, dez. 2005].

Moreira, J.; Penafiel, A. A contribution to the study of dementia paralytica in Brazil. Journal of Mental Science, v. 53, p. 507-521, 1907. [Traduzido e publicado como: Moreira, J.; Penafiel, A. Contribuição ao estudo da dementia paralytica no Brasil. Revista Latinoamericana de Psicopatologia Fundamental, v. 8, n. 4, p. 812-827, dez. 2005].

Rev. Latinoam. Psicopat. Fund., São Paulo, v. 13, n. 2, p. 318-332, junho 2010 
Moreira se correspondeu com Kraepelin sobre seus planos de visitar o Brasil, junto com A. Alzheimer, para uma expedição de pesquisa (veja-se a correspondência em Dalgalarrondo, 1996). Tal expedição não aconteceu, segundo Moreira, porque Kraepelin teria se desapontado diante da informação de que aqui não encontraria indígenas reunidos em número significativo para a realização de um estudo psiquiátrico comparativo, nos moldes em que vinha planejando (Moreira, 1926).

\section{Brevíssima história da paranoia}

Em 1906, Moreira e Peixoto apresentaram ao XV Congresso Internacional de Medicina de Lisboa o trabalho intitulado "La paranoïa légitime: son origine et nature" (Moreira, 1908; Peixoto, 1911]), uma versão do citado artigo "A paranoia e as síndromes paranoides" (1905a). O título do trabalho explicita o ponto central do debate; afinal, o que seria a "paranoia legítima" ou verdadeira? ${ }^{10}$ Ou seja, a qual quadro clínico deveria ser reservado esse nome, ou antes, que manifestações psicopatológicas poderiam ser agrupadas sob esta rubrica, de maneira que o nome paranoia indicasse uma entidade nosológica de contornos delimitados, que fosse útil para os fins da clínica psiquiátrica.

A questão colocada pelos médicos brasileiros não era perfunctória, já que, como observara outro autor nacional, "os delírios sistematizados e a acepção da palavra "paranoia' fizeram emergir profundas divergências entre os psiquiatras" (Nina-Rodrigues, 2009, p. 767 [1902]). A história da paranoia é longa e se insere na história dos quadros delirantes, ou melhor, começa com a delimitação do moderno conceito de delírio (Berrios, 1998), sendo que o leitor interessado pode recorrer a estudos específicos. ${ }^{11}$

10. De forma original, Mário Eduardo Costa Pereira (2007) indicou que, em 1932, Jacques Lacan mencionará esse trabalho de Moreira e Peixoto em sua tese (Sobre a psicose paranoica em suas relações com a personalidade), como uma das referências para sustentar a necessidade de demarcar a "paranoia legítima", em relação aos demais quadros paranoides. Segundo Pereira, nas palavras de Lacan, quanto à definição de paranoia reinava "uma diversidade digna de Babel".

11. Tais como a clássica revisão de Aubrey Lewis (1970); o artigo de Ian Dowbiggin (2000), investigação sobre a história conceitual e social da paranoia, que chega à atual "era do DSM"; os capítulos de Kenneth Kendler e de Ian Dowbiggin sobre os transtornos delirantes (delusional disorders) no livro de Berrios e Porter (1999); o capítulo sobre a paranoia de George Lanteri-Laura e Martine Gros em Nouvelle histoire de la psychiatrie (1983) e o fundamental capítulo sobre os delírios no livro de German Berrios (1998). 


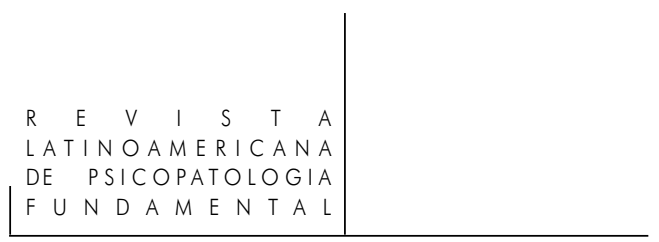

Observa Dowbiggin (2000, p. 43) que, desde meados do século XIX, a chamada "questão da paranoia" envolveu os mais conceituados alienistas alemães, e a lista dos debatedores parece uma espécie de "who is who" da psiquiatria germânica: Griesinger, Meynert, Schule, Wernicke, Krafft-Ebing, Arndt, Mendel, Neisser, Kraepelin etc. Depois de 1890, há entre eles certa concordância no uso do termo paranoia (ou Verrücktheit) como designação genérica das loucuras delirantes crônicas, em que não havia alterações de consciência e tampouco marcantes perturbações formais do pensamento. ${ }^{12}$

No restante da Europa, a paranoia ocupava cada vez mais lugar destacado nas controvérsias nosológicas ${ }^{13}$ (Kendler, 1999; Dowbiggin, 1999). Os italianos adotavam a palavra como designação ampla, pelo menos desde a década de 1880; na França, desde Esquirol, os alienistas vinham descrevendo e analisando os delírios sistematizados autorreferentes (de perseguição, de grandeza, místicos, eróticos etc.) e, com poucas exceções, rejeitavam o uso do termo paranoia, por julgá-lo demasiado impreciso (Lewis, 1970).

É a esta história que se refere Kraepelin nas primeiras páginas do texto aqui republicado, ao criticar a demasiada abrangência dada pelos autores alemães ao termo paranoia, a ponto de, em sua opinião, ser clinicamente inútil usar o diagnóstico de paranoia, se ele referia-se a quase $80 \%$ dos doentes internados nos hospícios alemães.

\section{A paranoia na sétima edição (1904) do Lehrbuch de Kraepelin}

Não caberia nesta apresentação detalhar o desenvolvimento da posição da paranoia nas classificações anteriores de Kraepelin, ou seja, da primeira (1883)

12. Como recorda Pereira (2007), no estudo sobre o caso Schreber, de 1911, Sigmund Freud utiliza os termos paranoia, dementia paranoides e diagnósticos correlatos referindo-se às sexta (1899) e sétima (1904) edições do tratado de Kraepelin.

13. Na mesma linha do "who is who", se tomarmos apenas dois textos de autores brasileiros que revisaram a história da paranoia, em inícios do século XX (Nina-Rodrigues, 1902; e Moreira e Peixoto, 1905a), encontraremos citados, entre outros: Bénédict-Augustin Morel, Carl Westphal, Charles Lasègue, E. Mendel, Emil Kraepelin, Enrico Morselli, Eugenio Tanzi, Evariste Marandon de Montyel, F. Del Grecco, Gilbert Ballet, H. Schüle, Henry Maudsley, Jacques Roubinovitch, Jules Falret, Jules Séglas, Júlio de Mattos, Maurice Legrain, Paul Garnier, P. Keraval, Richard von Krafft-Ebing, Theodor Meynert, Théodule Ribot, Valentin Magnan, Wilhelm Sander e Wilhelm Weygandt. 
à sexta (1899) edições de seu tratado. ${ }^{14}$ Resumidamente, pode-se apontar que na quinta edição (1896), ele alocou a Verrücktheit no grande grupo das doenças mentais constitucionais, o mesmo da loucura periódica; e, como parte do grupo das doenças adquiridas, descreveu três tipos de processos de enfraquecimento mental (Verblödungsprocesse): a dementia praecox, a catatonia, e a dementia paranoides. Na sexta edição (1899), Kraepelin juntou os Verblödungsprocesse na entidade dementia praecox (nas formas clínicas hebefrênica, catatônica e paranoide ou dementia paranoides); a paranoia permaneceu como entidade de origem constitucional, exemplar no contraste com a forma paranoide da demência precoce, em termos que seguirão similares na edição de 1904 (Bercherie, 1989).

Nessa edição, a discussão sobre a paranoia segue os princípios gerais enunciados no prefácio do tratado, onde Kraepelin dizia que

... uma vez que até agora, nem na anatomia patológica, nem na etiologia, nem na clínica as pesquisas avançaram o bastante para nos fornecer um fundamento seguro com vistas à classificação dos distúrbios mentais, devemos, para levar a cabo nossa obra, utilizar os recursos que forem colocados à nossa disposição por estes três lados. (Kraepelin, 1904, apud Bercherie, 1989, p. 168)

Desta maneira, o autor propõe usar como critérios de inclusão de certos quadros clínicos em uma mesma entidade mórbida: a indicação (ou suposição) da existência de causas comuns, suas marcha e duração e seu desfecho. Considerando que delírios, ilusões e alucinações poderiam ocorrer em várias formas de alienação, e assim não eram bons recursos de discriminação diagnóstica, Kraepelin afirma que o único meio efetivo para esclarecer as peculiaridades das enfermidades mentais seria fixar a atenção no andamento da moléstia e no seu desenlace. Toma a paralisia geral progressiva (sifilítica, então de agente etiológico desconhecido) como modelo de enfermidade em que o conhecimento acumulado sobre a evolução do quadro clínico típico permitia a razoável previsão do término fatal.

Assim, Kraepelin diz reservar o nome de paranoia para "um grupo de casos nos quais as concepções delirantes (Wahnvorstellung), se bem que não constituam exclusivamente o quadro clínico, são todavia o indício mórbido que mais se sobressai. Neste grupo costuma-se desenvolver um inabalável sistema delirante lento e duradouro, com completa conservação do discernimento, clareza e ordem na associação do pensamento, da vontade e da ação”. Acrescenta ainda que a pa-

14. Neste sentido, veja-se Paul Bercherie (1989), que faz uma detalhada análise comparada de oito edições do tratado de Kraepelin, sendo que a trajetória da paranoia na nosologia kraepeliniana pode ser acompanhada neste cuidadoso trabalho, bem como suas relações com os chamados delírios sistematizados dos autores franceses. 


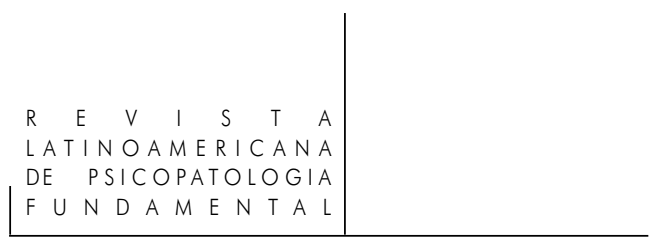

ranoia conduz "necessariamente a uma completa modificação em toda a maneira de apreciar a vida, a uma loucura de posição (Verrükung)" e que o desenvolvimento da moléstia "parece executar-se sempre com muita lentidão" (Kraepelin, 1905, p. 103).

De acordo com ele, a paranoia não compromete a personalidade, somente produz uma apreciação mórbida do mundo, o que explicaria as características dos delírios dos paranoicos, sua coerência e consistência internas e sua plausibilidade, bem como seus atos aparentemente pautados pela lógica racional. Ao contrário, diz ele, na demência precoce as ideias delirantes são bizarras e mutáveis e o pensamento confuso, além de haver enfraquecimentos da vontade e do raciocínio, bem como alterações sensoperceptivas variadas e sensações de influência externa. ${ }^{15}$ A raridade de alucinações, ou sua fraca intensidade, é apontada como contrastante com o que ocorreria na demência precoce. ${ }^{16}$

Finalizando, Kraepelin discute detalhes do diagnóstico diferencial da paranoia, destacando a diferenciação a ser feita com as formas delirantes da demência precoce; observa que ao paranoico falta a perturbação da vontade, perturbação fundamental na demência precoce (e expressa por manifestações como negativismo, estupor, estereotipia e excitação, automatismo e impulsividade). Recorda também o diagnóstico diferencial necessário com certas formas de epilepsia e com o "delírio dos bebedores", ressaltando que a observação continuada de cada caso elucidaria a que categoria este deveria pertencer.

\section{As ideias de Kraepelin no século XX: fastígio, descenso e "redescoberta"}

Citando o inglês Havelock Ellis, os editores dos Arquivos Brasileiros de Psiquiatria assinalam que Emil Kraepelin era "the greatest living master of scientific psychiatry" (Moreira e Peixoto, 1905a; Moreira, 1919). No mesmo sentido, no necrológio de Kraepelin, diz Moreira (1926) que Munique tornara-se a Meca para onde afluíam psiquiatras de todo o mundo, em busca de orientação do mestre alemão. No entanto, depois de atingir seu ápice nas primeiras três décadas do século XX, a sua popularidade entre os psiquiatras entra em franco declínio, principalmente após a Segunda Guerra Mundial e até a década de 1970. Então,

15. Sobre a demência precoce, veja-se Kraepelin, 1905.

16. $\mathrm{Na}^{\mathrm{a}}$ edição do tratado, Kraepelin considerará que alucinações verdadeiras não existiam na paranoia, alocando os casos em que estas eram evidentes na nova categoria parafrenia, que surge como intermediária entre a demência precoce e a paranoia (Kendler, 1999) quanto ao grau de preservação do pensamento, do afeto e da vontade durante o curso da doença (Lewis, 1970). 
ela renascerá com os autodenominados neokraepelinianos, no contexto dos debates sobre a cientificidade da psiquiatria e de suas classificações, nos EUA da década de 1980 (Engstrom e Weber, 2007; Jablensky, 2007).

Assim, nas palavras de Engstrom e Weber, o médico alemão torna-se uma espécie de pai ou patrono pós-freudiano da psiquiatria clínica, da terceira edição do Diagnostic and Statistical Manual of Mental Disorders (DSM-III, da Associação Psiquiátrica Americana) e dos métodos quantitativos de avaliação (e de quantas coisas mais se queira que ele seja fundador), e à sua obra passam a se endereçar as questões mais prementes da psiquiatria de fins do século XX e inícios do XXI. Demarcada em exagerada contraposição a Freud, esta nova posição em que se coloca a obra de Kraepelin parece fundada em uma interpretação demasiado anacrônica e limitada da história da psiquiatria.

Asseveram Berrios e Hauser (1988) que os psiquiatras ainda vivem em um mundo kraepeliniano. E, completa Jablensky (2007), se muito do discurso psiquiátrico atual é moldado pela visão nosológica de Kraepelin, nem sempre esta é mencionada de forma acertada. Uma das características mais marcantes de algumas equivocadas invocações ao nome do mestre alemão é o seu sensível anacronismo (Engstrom e Weber, 2007); deste modo, por exemplo, alguns psiquiatras contemporâneos não se inibem em estabelecer ligações diretas entre o pensamento de Emil Kraepelin e concepções e práticas que lhe são totalmente extemporâneas, sejam pesquisas recentes sobre a neurobiologia do transtorno afetivo bipolar ou a mania nos tempos hipocráticos.

Obviamente, a original, extensa e multifacetada obra de Emil Kraepelin deve ser compreendida em seu específico contexto de produção, e tal compreensão somente pode ser obtida por meio de pesquisas que analisem diferentes dimensões históricas do período em que viveu esse médico alemão, tendo em conta suas premissas científicas, culturais, filosóficas e políticas, suas atividades como clínico e pesquisador, as características das instituições científicas em que trabalhou, sua inserção nos meios profissionais etc. ${ }^{17}$ Uma área de pesquisas

17. Neste sentido, veja-se o número especial de History of Psychiatry dedicado a estudos sobre Emil Kraepelin, organizado por Eric Engstrom e Matthias Weber (vol. 18, n. 3, 2007). Uma apreciação geral da trajetória intelectual de Kraepelin, com ênfase nos vetores que guiaram sua agenda de pesquisa, pode ser lida no artigo de Engstrom (2007).

Há reedições recentes das principais obras de Kraepelin; em inglês, destaca-se a coleção Lifetime Editions of Kraepelin in English (5 volumes, Bristol: Thoemmes Press, 2002); em espanhol, há edições de alguns de seus trabalhos pela editora Polemos e, em português, pela editora Climepsi. Em alemão, a edição crítica dos escritos de Kraepelin (documentos diversos e correspondência) tem sido levada a cabo pelo Instituto Max Planck de Psiquiatria. 
promissora é aquela que se volta à recepção e difusão das ideias de Kraepelin em conjunturas específicas, por exemplo, em países da Europa e das Américas (Engstrom e Weber, 2007), tema que se pretendeu apenas esboçar aqui.

Da mesma forma, buscou-se apresentar a categoria paranoia em um dado momento histórico do processo de construção da nosologia kraepeliniana, indicando que se trata de focalizar uma etapa de um trabalho em curso. A análise histórica diacrônica mostra as muitas hesitações de Kraepelin quanto à definição e à posição da paranoia, como observou Lewis (1970), mas é bom lembrar que aqui tratamos de um texto de 1904, em que a paranoia parece ao nosso autor uma razoável candidata a entidade mórbida, tanto quanto (ou quiçá um pouco menos que) a demência precoce.

Como se sabe, nas duas classificações psiquiátricas internacionais hoje vigentes, a CID 10 (OMS, 2003 [1993]) e o DSM-IV (APA, 1994), a paranoia foi expungida da nomenclatura principal, restando incorporada à rubrica dos transtornos delirantes (delusional disorders). O que não se apaga facilmente, entretanto, são as elucidações que nos traz a história da paranoia sobre este fascinante jogo que se chama diagnosticar em psiquiatria.

\section{Referências}

American Psychiatric Association (APA). Diagnostic and Statistical Manual of Mental Disorders. $4^{\text {th }}$ edition (DSM IV). Washington: American Psychiatric Association, 1994.

Bercherie, P. Os fundamentos da clínica: história e estrutura do saber psiquiátrico. Tradução Vera Ribeiro. Rio de Janeiro: Jorge Zahar, 1989.

Berrios, G. E. Delusions. In: Berrios, G. E. The history of mental symptoms: descriptive psychopathology since the nineteenth century. Cambridge: Cambridge University Press, 1998. p. 85-139.

Berrios, G. E.; Hauser, R. The early development of Kraepelin's ideas on classification: a conceptual history. Psychological Medicine, v. 18, n. 4, p. 813-821, 1988.

Berrios, G. E.; Porter, R. (Org.). History of Clinical Psychiatry: The Origin and History of Psychiatric Disorders. London: Athlone, 1999.

Dalgalarrondo, P. Civilização e loucura: uma introdução à história da etnopsiquiatria. São Paulo: Lemos, 1996.

Dowbiggin, I. Delusional diagnosis? The history of paranoia as a disease concept in 
the modern era. History of Psychiatry, v.11, n. 41, p. 37-69, 2000. Disponível em: <http://hpy.sagepub.com/cgi/reprint/11/41/037>. Acesso em: 10 nov. 2009.

Dowbiggin, I. Delusional disorder: social section. In: Berrios, G. E.; Porter, R. (Org.). History of Clinical Psychiatry: The Origin and History of Psychiatric Disorders. London: Athlone, 1999. p. 372-383.

Engstrom, E. J. (1908). "On the question of degeneration” by Emil Kraepelin. History of Psychiatry, v. 18, n. 3, p. 389-404, 2007. Disponível em: <http://hpy.sagepub.com/ cgi/content/abstract/18/3/389>. Acesso em: 10 nov. 2009.

Engstrom, E. J.; Weber, M. M. Making Kraepelin history: a great instauration? History of Psychiatry, v. 18, n. 3, p. 267-273, 2007. Disponível em: <http:// hpy.sagepub.com/cgi/content/abstract/18/3/267>. Acesso em: 10 nov. 2009.

JABLENSKY, A. Living in a Kraepelinian world: Kraepelin's impact on modern psychiatry. History of Psychiatry, v. 18, n. 3, p. 381-388, 2007. Disponível em: <http:// hpy.sagepub.com/cgi/content/abstract/18/3/381>. Acesso em: 10 nov. 2009.

Kendler, K. S. Delusional disorder: clinical section. In: Berrios, G. E.; Porter, R. (Org.). History of Clinical Psychiatry: The Origin and History of Psychiatric Disorders. London: Athlone, 1999. p. 360-371.

KRAEPELIN, E. (1887). The directions of psychiatric research (1887). (“Classic Text No. 63”, tradução e notas de E. J. Engstrom e M. M. Weber). History of Psychiatry, v. 16, n. 3, p. 350-364, 2005.

. (1904). Paranoia (Verrücktheit). Traduzido [por A. Austregésilo] da obra do Prof. Emil Kraepelin, Die Psychiatrie, Leipzig, 1904, v. 2, p. 590. [Psychiatrie: Ein Lehrbuch für Studierende und Ärzte. Siebente, vielfach umgearbeitete Auflage. II. Band (in 2 Teilbänden). Klinische Psychiatrie. Barth Verlag: Leipzig. 1904]. Arquivos Brasileiros de Psiquiatria, Neurologia e Ciências Afins, Rio de Janeiro, v. 1, n. 1, p. 98-106; v. 1, n. 2, p. 183-196; v. 1, n. 3-4, 301-309, 1905. . (1905). Introdução à psiquiatria clínica. Terceira lição: demência precoce. Revista Latinoamericana de Psicopatologia Fundamental, São Paulo, v. 4, n. 4, p. 130-137, dez. 2001. Disponível em: <http:/www.fundamentalpsychopathology. org/art/dez1/classicos.kraepelin.pdf>. Acesso em: 10 nov. 2009.

Lanteri-Laura, G; Gros, M. La paranoïa. In: Postel, J.; Quétel, C. (Org.). Nouvelle histoire de la psychiatrie. Toulouse: Privat, 1983. p. 334-341.

Lewis, A. Paranoia and paranoid: a historical perspective. Psychological Medicine, v. 1, n. 1, p. 2-12, 1970.

Moreira, J. Psiquiatria. In: Academia Nacional de Medicina (Org.). Em comemoração do centenário do ensino médico. Rio de Janeiro: Tipografia do Jornal do Commercio, 1908. p. 433-448. 


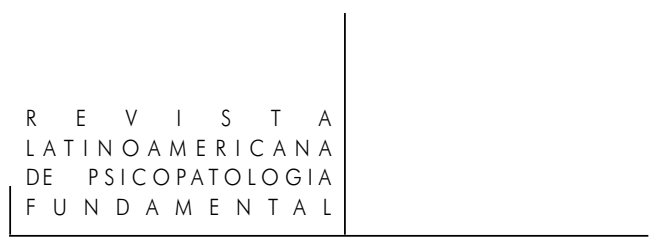

Classificações em medicina mental. Arquivos Brasileiros de Neuriatria e Psiquiatria, Rio de Janeiro, v. 1, n. 1, p. 93-115, 1919.

. O novo agrupamento nosográfico das doenças mentais do prof. Emil

Kraepelin. Arquivos Brasileiros de Neuriatria e Psiquiatria, Rio de Janeiro, v. 3, n.12, p. 181-189, 1921. [Traduzido de Einführung in die psychiatrische Klinik, 1921].

Professor Emílio Kraepelin. Arquivos Brasileiros de Neuriatria e Psiquiatria, Rio de Janeiro, v. 8, n. 3-4, p. 131-138, 1926.

Moreira, J.; Рeixoto, A. A paranoia e as síndromes paranoides. Arquivos Brasileiros de Psiquiatria, Neurologia e Ciências Afins, Rio de Janeiro, v. 1, n. 1, p. 5-33, 1905 a.

. Classificação das moléstias mentais do professor Emil Kraepelin.

Arquivos Brasileiros de Psiquiatria, Neurologia e Ciências Afins, Rio de Janeiro, v. 1, n. 2, p. 204-216; v. 1, n. 3-4, p. 310-316, 1905 b.

Morel, P. Dicionário biográfico PSI. Tradução Lucy Magalhães. Rio de Janeiro: Jorge Zahar, 1997. p. 140-141.

NinA-Rodrigues, R. (1902). Atavismo psíquico e paranoia. Revista Latinoamericana de Psicopatologia Fundamental, São Paulo, v. 12, n. 4, p. 766-789, dez. 2009. Disponível em: 〈http://www.scielo.br/pdf/rlpf/v12n4/v12n4a12.pdf>. Acesso em: 10 fev. 2010.

Oda, A. M. G. R.; Dalgalarrondo, P. A paranoia, segundo Juliano Moreira e Afrânio Peixoto. Revista Latinoamericana de Psicopatologia Fundamental, São Paulo, v. 4, n. 2, p. 125-133, jun. 2001.

Oda, A. M. G. R.; Dalgalarrondo, P.; Banzato, C. E. M. Some origins of crosscultural psychiatry. History of Psychiatry, v. 16, n. 2, p. 155-169, 2005. Disponível em: <http://hpy.sagepub.com/cgi/reprint/16/2/155>. Acesso em: 1 maio 2010.

OdA, A. M. G. R.; Piccinini, W. Dos males que acompanham o progresso do Brasil: a psiquiatria comparada de Juliano Moreira e colaboradores. Revista Latinoamericana de Psicopatologia Fundamental, São Paulo, v. 8, n. 4, p. 788-793, dez. 2005. Disponível em: 〈http://www.fundamentalpsychopathology.org/art/v08_04/historia.pdf〉.

Acesso em: 1 maio 2010.

Organização Mundial da Saúde (OMS). Classificação estatística internacional de doenças e problemas relacionados à saúde. 10. revisão (CID 10). Transtornos mentais e comportamentais, doenças do sistema nervoso (separata). Tradução da edição em inglês (1993). Centro Colaborador da OPAS/OMS para classificação de doenças em português. São Paulo: Edusp. 2003.

Peiхoтo, А. (1911). Medicina Legal: psicopatologia forense (vol. 2). 5. ed. Rio de Janeiro: Francisco Alves, 1938. p. 282.

Pereira, M. E. C. Lacan com Juliano Moreira e Afrânio Peixoto: a autofilia primitiva, o narcisismo e a questão da paranoia legítima. In: Associação Psicanalítica de Porto Alegre (Org.). Psicose: aberturas da clínica. Porto Alegre: Libretos, 2007. p. 18-53. 
SAurí, J. J. O que é diagnosticar em psiquiatria. São Paulo: Escuta. 2001.

SHORTER, E. A historical dictionary of psychiatry. Oxford: Oxford University Press, 2005. p. 156-157.

Teive, H. A. G.; Sá, D.; Silveira Neto, O.; Silveira, O. A. da; Werneck, L. C. Professor Antonio Austregésilo: o pioneiro da neurologia e do estudo dos distúrbios do movimento no Brasil. Arquivos de Neuro-Psiquiatria, v. 57, n. 3B, p. 898-902, 1999. Disponível em: <http://www.scielo.br/pdf/anp/v57n3B/1209.pdf>. Acesso em: 1 maio 2010.

\section{Resumos}

(Paranoia in 1904 - a stage in Emil Kraepelin's construction of this nosology)

This article introduces Kraepelin's text on paranoia, taken from the seventh edition of his Treatise on Psychiatry (1904), translated into the Portuguese and published for the first time in 1905 in Revista Brasileira de Psiquiatria, neurologia e ciências afins (Brazilian Journal of Psychiatry, Neurology and Related Sciences. The article indicates the place of paranoia in Kraepelin's nosology and discusses aspects of the diffusion of his ideas in Brazil.

Key words: Emil Kraepelin, paranoia - history, psychiatric nosology - history, delirious disorders - history, Brazilian Archives in Psychiatry, Neurology and Related Sciences

(La paranoïa en 1904 - une étape de la construction nosologique d'Emil Kraepelin)

Cet article nous présente le texte de Kraepelin sur la paranö̈a, extrait de la septième édition de son manuel de psychiatrie (1904), traduit en portugais et publié en 1905 dans la revue Archives Brésiliennes de Psychiatrie, Neurologie et des Sciences Connexes. On indique la position de cette catégorie dans la nosologie de Kraepelin et discute les aspects de la diffusion de ses idées au Brésil.

Mots clés: Emil Kraepelin, Paranoïa - histoire, Nosologie Psychiatrique - histoire, Troubles Délirants - histoire, Archives Brésiliennes de Psychiatrie, Neurologie et Sciences Connexes

(La paranoia en 1904 - una etapa de la construción nosologica de Emil Kraepelin)

Este artículo presenta el texto de Kraepelin sobre la paranoia, extraído de la séptima edición de su tratado de psiquiatría (1904), traducido para el portugués y publicado por la primera vez en 1905, en la revista Arquivos Brasileiros de Psiquiatria, Neurologia e Ciências Afins. Se indica en él, la posición de esta categoría en la 


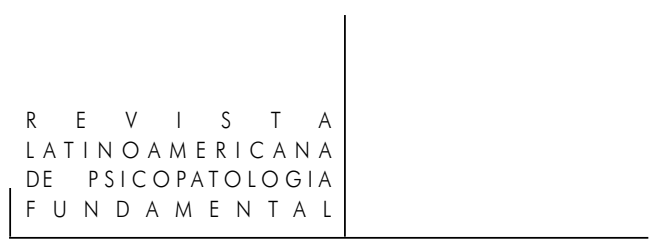

nosología de Kraepelin, así como se discuten aspectos de la difusión de sus ideas en el Brasil.

Palabras claves: Emil Kraepelin, paranoia - historia, nosología psiquiátrica - historia, trastornos delirantes - historia, Archivos Brasileños de Psiquiatría, Neurología y Ciencias Afines

Citação/Citation: OdA, A.M.G. R. A paranoia em 1904 - uma etapa na construção nosológica de Emil Kraepelin. Revista Latinoamericana de Psicopatologia Fundamental, São Paulo, v. 12, n. 3, p. 318-332, jun. 2010.

Editor do artigo/Editor: Profa. Dra. Ana Maria Galdini Raimundo Oda; Prof. Dr. Paulo Dalgalarrondo

Recebido/Received: $10.5 .2010 /$ 5.10.2010 Aceito/Accepted: 15.5.2010 / 5.15.2010

Copyright: @ 2009 Associação Universitária de Pesquisa em Psicopatologia Fundamental/ University Association for Research in Fundamental Psychopathology. Este é um artigo de livre acesso, que permite uso irrestrito, distribuição e reprodução em qualquer meio, desde que o autor e a fonte sejam citados/This is an open-access article, which permits unrestricted use, distribution, and reproduction in any medium, provided the original author and source are credited.

Financiamento/Funding: A autora declara não ter sido financiada ou apoiada/The author has no support or funding to report.

Conflito de interesses/Conflict of interest: A autora declara que não há conflito de interesses/The author declares that has no conflict of interest.

\section{Ana Maria Galdini Raimundo Oda}

Médica psiquiatra, com mestrado em Saúde Mental (1998) e doutorado em Ciências Médicas (2003) pela Universidade Estadual de Campinas - Unicamp (Campinas, SP, Brasil); tem experiência nas áreas de psiquiatria clínica e de saúde mental em saúde coletiva, e ainda em pesquisa em história da medicina, da psiquiatria e da psicopatologia; editora associada da Revista Latinoamericana de Psicopatologia Fundamental (seção História da Psiquiatria) e atual coordenadora do Departamento de História da Psiquiatria da Associação Brasileira de Psiquiatria; professora adjunta do Departamento de Medicina do Centro de Ciências Biológicas e da Saúde da Universidade Federal de São Carlos - UFSCar (São Carlos, SP, Brasil). Universidade Federal de São Carlos - CCBS - Departamento de Medicina Rodovia Washington Luis, Km 235

13565-905 São Carlos, SP, Brasil anaoda@ufscar.br 